\title{
Topical ganciclovir in the treatment of acute herpetic keratitis
}

This article was published in the following Dove Press journal:

Clinical Ophthalmology

5 August 2010

Number of times this article has been viewed

\author{
Khalid F Tabbara ${ }^{1,2,3}$ \\ Noorjehan Al Balushi' \\ 'The Eye Center and The Eye \\ Foundation for Research in \\ Ophthalmology, Riyadh, ${ }^{2}$ Department \\ of Ophthalmology, College of \\ Medicine, King Saud University, \\ Riyadh, Kingdom of Saudi Arabia; \\ ${ }^{3}$ The Wilmer Ophthalmological \\ Institute of The Johns Hopkins \\ University School of Medicine, \\ Baltimore, Maryland, USA
}

Abstract: Herpetic keratitis is caused by herpes simplex virus (HSV) and is a common cause of corneal blindness. Following a primary ocular herpetic infection, latency of the virus occurs, followed by subsequent recurrences of herpetic keratitis. Such recurrences may lead to structural damage of the cornea. Recurrent herpetic keratitis is a common indication for corneal transplantation. Recurrences of herpetic keratitis in the corneal graft may lead to corneal graft rejection. Several antiviral agents for HSV are available, including the thymidine analogs. Prolonged use of thymidine analogs may lead to toxicity of the ocular surface, including epithelial keratitis, corneal ulcers, follicular conjunctivitis, and punctal occlusions. Availability of topical antiviral agents that are safe and effective in the treatment and prophylaxis of herpetic keratitis is highly desirable. Ganciclovir is a potent inhibitor of members of the herpes virus family. The drug has been used systemically for the treatment of cytomegalovirus (CMV) retinitis. Its hematologic toxicity secondary to systemic administration led to its limited use in herpetic infections. On the other hand, topical ganciclovir has been shown to be as safe and effective as acyclovir in the treatment of herpetic epithelial keratitis. Furthermore, topical ganciclovir can reach therapeutic levels in the cornea and aqueous humor following topical application. Several clinical trials have shown that topical ganciclovir $0.15 \%$ ophthalmic gel is safe and effective in the treatment and prophylaxis of herpetic epithelial disease. Long-term use of ganciclovir ophthalmic gel in patients with penetrating keratoplasty following herpetic keratitis has prevented recurrences of the disease. Topical ganciclovir ophthalmic gel is well tolerated, does not cause toxic effects on the ocular surface, and does not cause hematologic abnormalities. Clinical studies have underscored the potential role of ganciclovir ophthalmic gel in the treatment and prophylaxis of herpetic epithelial keratitis. Future randomized, controlled, multicenter, prospective clinical trials are needed to assess the long-term safety and efficacy of topical ganciclovir in the treatment and prevention of herpetic keratitis and uveitis.

Keywords: herpetic keratitis, cornea, herpes simplex, ganciclovir, acyclovir

\section{Introduction}

The ocular and adnexal structures are frequently the target of infection by herpes simplex virus (HSV). HSV is involved in the pathogenesis of several ocular disorders and may lead to blepharitis, vesicular dermatitis of the eyelids, conjunctivitis, keratitis, trabeculitis, anterior uveitis, acute retinal necrosis syndrome, retinitis, and optic neuritis (Table 1). Herpetic corneal epithelial infections are by far the most common ocular manifestation. Herpetic ocular disease may be primary, latent, or recurrent (secondary). ${ }^{1-3}$ Ocular infection in a patient who is not immune to the virus and not previously infected is referred to as primary disease. Patient may develop viral latency
Correspondence: KF Tabbara

The Eye Center, PO Box 55307, Riyadh I 1534, Kingdom of Saudi Arabia

Tel +966 I464 96। 4

Fax $+966 \quad 14629675$

Email k.tabbara@nesma.net.sa 
Table I Ocular manifestations of herpes simplex of the eye and adnexa

\begin{tabular}{l} 
Eyelid \\
Dermatitis and vesicular eruption and ulceration \\
Punctal occlusion \\
Conjunctiva \\
Follicular conjunctivitis \\
Membranous conjunctivitis \\
Conjunctival cicatrization \\
Episclera/sclera \\
Episcleritis \\
Scleritis \\
Cornea \\
A. Epithelium \\
Dendritic or geographic ulcer \\
B. Stromal \\
Necrotizing keratitis \\
Non-necrotizing keratitis \\
Disciform keratitis \\
Interstitial keratitis \\
C. Endothelium \\
Endotheliitis \\
D. Corneal complications \\
Corneal vascularization, corneal scarring \\
Trophic keratitis \\
Lipid keratopathy \\
Corneal perforation \\
Postinflammatory corneal ectasia \\
Acute anterior granulomatous or nongranulomatous uveitis \\
Trabeculitis \\
Secondary glaucoma \\
Iris atrophy \\
Iridocyclitis and trabeculitis \\
Uvea nasculitis neritis \\
\hline
\end{tabular}

after a primary infection and the virus stays dormant in the nerve ganglia. It may later cause recurrent clinical manifestations. Ocular infections in adults are predominantly due to HSV Type 1. Genito-ocular spread can occur and may be caused by HSV Type 2, but is less common. The disease may also be related to postnatal infection with HSV Type 1. An intrauterine- or neonatal birth canal-acquired infection with HSV Type 2 may occur. In herpes-infected neonates and infants without central nervous system involvement, keratitis occurs in $80 \%$ of cases and conjunctivitis occurs in approximately $10 \%$ of cases. Visual loss in ocular herpetic infection may be due to keratitis, uveitis, cataract, or glaucoma, while in neonatal herpetic infections the visual loss is commonly due to optic atrophy and chorioretinitis. Following a primary infection, the disease may recur in the cornea and may result in epithelial keratitis. Recurrent epithelial keratitis may stay in the epithelial layer or may involve the deeper stromal layer and endothelium.

Risk factors for HSV reactivation are well known and include stress, exposure to ultraviolet radiation, menstruation, fever, use and abuse of corticosteroids, and trauma including surgical trauma. ${ }^{4,5}$ The disease is usually unilateral. Bilateral herpetic keratitis may occur in patients with systemic diseases, such as atopic disorders, malignancy, debilitating illnesses, or in patients on immunosuppressive agents. ${ }^{2}$

Herpetic keratitis is a common cause of ocular morbidity and represents the most common cause of corneal blindness. ${ }^{1}$ Recurrent herpetic keratitis is also a common indication for corneal transplantation. Recurrences of herpetic epithelial keratitis may occur in the corneal graft and result in structural damage of the cornea, leading to corneal graft rejection and failure. The ocular disease could take one of two forms. The first is the primary ocular disease occurring in a patient who has no previous exposure to herpes virus, and the second is latent disease in patients who have dormant HSV in the nerve ganglia. Primary herpetic disease can be clinical or subclinical. Viral reactivation in latency may lead to involvement of the lids, conjunctiva, cornea, uvea, retina, and/or optic nerve. Recurrent disease of the cornea is the most common, occurs in the latent phase, and may be epithelial, stromal, endothelial, or disciform.

There are several antiviral agents that are effective against HSV, and these include idoxuridine, vidarabine, trifluridine, acyclovir, famciclovir, valganciclovir, valacyclovir, and cidofovir. Idoxuridine and trifluridine act as thymidine analogs by formation of defective viral DNA molecules due to inhibition of DNA polymerase. ${ }^{3}$ Vidarabine, a purine analog, inhibits viral DNA polymerase and is incorporated by the viral DNA molecule. Idoxuridine, trifluridine, and vidarabine cause corneal epithelial toxicity because of their nonselective action on infected and noninfected host cell DNA molecules. Prolonged use of thymidine analogs, however, may cause ocular surface toxicity. Acyclovir is activated by HSV thymidine kinase to the monophosphate form and by cellular enzymes to acyclovir triphosphate, causing inhibition of Herpes virus DNA polymerase. Topical acyclovir is available as a 3\% ophthalmic ointment for the treatment of HSV corneal epithelial.

Several studies have shown that ganciclovir is highly effective in the inhibition of HSV Type 1 and Type 2. Systemic ganciclovir is indicated for sight-threatening CMV retinitis in severely immunocompromised people, CMV pneumonitis in bone marrow transplant recipients, prevention of CMV disease in bone marrow and solid organ transplant recipients, 
and confirmed CMV retinitis in people with acquired immunodeficiency syndrome (intravitreal implant).

The purpose of this paper is to provide a critical review of topical ganciclovir in the treatment of acute herpetic epithelial keratitis.

\section{Pharmacology and pharmacokinetics}

Ganciclovir (Figure 1) is an antiviral agent that is similar to acyclovir (Figure 2). Acyclovir is used for herpetic keratitis, whereas ganciclovir is used to treat or prevent cytomegalovirus (CMV) infections. Ganciclovir sodium is marketed under the trade names Cytovene ${ }^{\circledR}$ and Cymevene ${ }^{\circledR}$ (Roche). Ganciclovir for intraocular inserts is marketed under the trade name Vitrasert ${ }^{\circledR}$ (Bausch and Lomb). The device is approved for the treatment of CMV retinitis. A prodrug form with improved oral bioavailability (valganciclovir) is also available. Topical ganciclovir is available from Laboratoires Théa (Louis-Bleriot, Clermont-Ferrand, France) and is marketed under the names Virgan ${ }^{\circledR}$ and Zirgan ${ }^{\circledR}$ as $1.5 \mathrm{mg} / \mathrm{g}$ eye gel in tubes of $5 \mathrm{~g}$ each. The other ingredients in ganciclovir are carbomer (Carbopol ${ }^{\circledR}$ 974P), sorbitol, sodium chloride, and benzalkonium chloride.

Ganciclovir is a nucleoside analog that is selectively phosphorylated by virus-encoded thymidine kinase, and is subsequently phosphorylated by cellular enzymes. ${ }^{1}$ Ganciclovir is converted into ganciclovir monophosphate and then to ganciclovir diphosphate and finally, to ganciclovir triphosphate. Ganciclovir triphosphate inhibits Herpes virus DNA polymerase and arrests HSV replication. ${ }^{6-9}$ Following

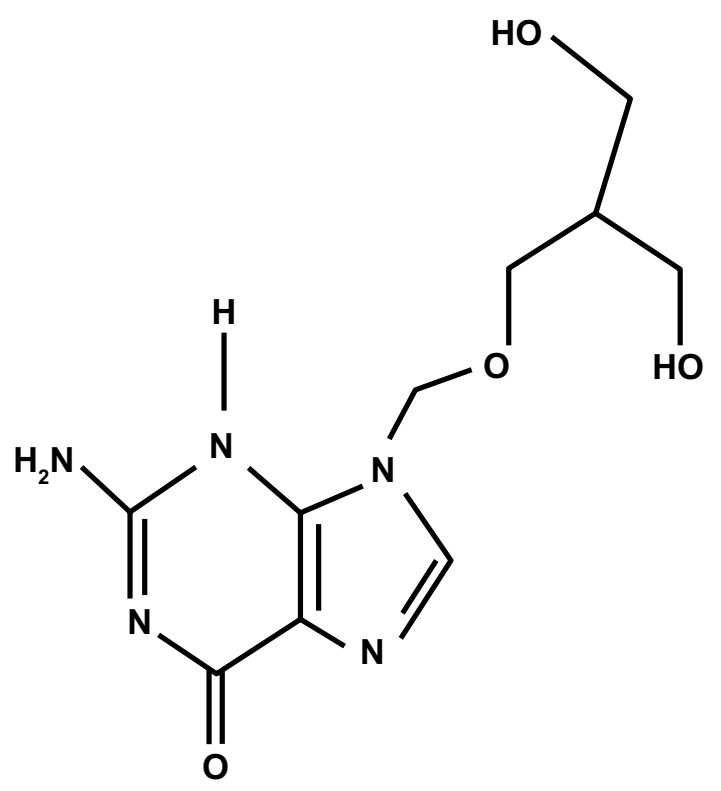

Figure I Structural formula of ganciclovir.

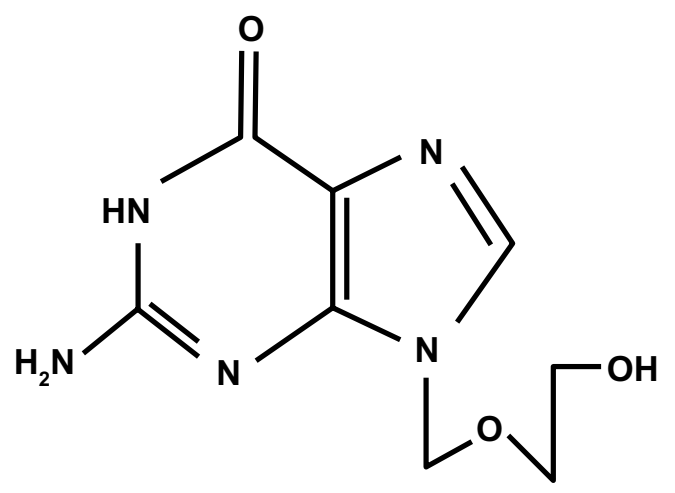

Figure 2 Structural formula of acyclovir.

topical application of ganciclovir, it has been shown that the drug can penetrate the corneal stroma and can reach the aqueous humor in therapeutic levels. ${ }^{9}$

In a study by Castela et al, ${ }^{9}$ ganciclovir ophthalmic gel was prepared in Carbomer 934P (NF XVII, Transphyto SA, Clermont-Ferrand, France). The drug-free vehicle was used as a placebo and the ganciclovir concentration levels of the tested gels were $0.0125 \%, 0.05 \%$, and $0.2 \%$. The investigators studied the effects of ganciclovir ophthalmic gel on HSV keratitis in rabbits. The efficacy of the three ganciclovir gel strengths were compared with placebo and 3\% acyclovir ophthalmic ointment using an HSV Type 1 rabbit keratitis animal model. All three ganciclovir concentrations showed clinical efficacy and significant reduction of corneal ulcer area, clouding, and vascularization $(P<0.05)$ and rapid inhibition of HSV shedding in tear films. The distribution of ganciclovir and acyclovir in the rabbit eyes were determined by high performance liquid chromatography. Both ganciclovir and acyclovir were found to reach higher levels in the cornea than in the aqueous humor. No ocular toxicity was noted in this study. Corneal penetration of ganciclovir was due to the small size of the ganciclovir molecule, its high lipophilicity, and its high cellular affinity resulting from its structural analogy with the endogenous nucleosides. This may explain the high diffusion of ganciclovir in the corneal epithelium (pKa 2.2 and 9.4 at $\mathrm{pH}$ 6.5-7.4). The destruction of the corneal epithelium by the virus allows penetration of the drug into the cornea so that it can reach the anterior chamber. Ganciclovir was found in the aqueous humor of healthy rabbits three hours after a series of six administrations of the gel formulation. ${ }^{9}$ The level of concentration was dependent on the ganciclovir dosage and frequency of application. Ganciclovir has poor water solubility, and its affinity for melanin in cells explains its preferential distribution in solid ocular tissues, such as the iris, compared with the aqueous 
humor. Ganciclovir was recovered from the aqueous humor four hours after the last topical administration of ganciclovir $0.2 \%$, and this could be due to the slow diffusion of the drug from the cornea to the aqueous humor. ${ }^{9}$

The physiochemical properties of acyclovir and ganciclovir are similar, and this explains the similarity in the ocular tissue distribution. The tissue concentrations are of the same order of magnitude when comparing ganciclovir ophthalmic gel $0.15 \%$ with $3 \%$ acyclovir ointment despite the difference in dosage strength. Furthermore, the plasma concentration four hours after the administration of ganciclovir gel was very low. This illustrates the limited plasma diffusion of the drug and its lack of systemic toxicity. With regard to the experimental design, one has to keep in mind that the rabbit model of herpetic keratitis is different from that of human ocular herpes. Extrapolation of the findings from the animal model to humans should be done with caution. The intraocular diffusion of ganciclovir may permit its use in the treatment of herpetic keratouveitis. The low plasma ganciclovir concentration after chronic gel application limits the risk of systemic toxicity. The efficacy of ganciclovir was observed in vitro and in vivo against HSV strains resistant to both idoxuridine and acyclovir. ${ }^{10}$

\section{Therapeutic interventions for HSV epithelial keratitis}

Wilhelmus ${ }^{11}$ performed a systematic review of the literature reported in the Cochrane Database. Surveys of ocular antiviral drugs for HSV eye disease were evaluated in a systematic review of all comparative clinical studies. The review revealed that antiviral agents are effective and nearly equivalent in their efficacy. The combination of a nucleoside antiviral agent with either debridement of the epithelium or with interferon seems to speed up healing of the epithelium. The studies that were reviewed did not consider the size of the lesion patients had prior to treatment. Debridement in combination with antiviral therapy appears to be more effective than antiviral chemotherapy alone, but this issue remains inconclusive. Removal of infected epithelial cells by debridement appears to be effective, but antiviral agents against HSV are needed to prevent recrudescence of epithelial keratitis. ${ }^{1}$ Antiviral agents did not increase the speed of healing when compared with debridement, but reduced the risks of recurrence of epithelial keratitis. Ganciclovir appear to be as effective as other antiviral agents in the treatment of herpetic keratitis. ${ }^{1}$

\section{Ganciclovir ophthalmic gel in herpetic keratitis}

Topical ganciclovir has been studied in animal models of herpetic keratitis and healthy volunteers, and in several prospective, randomized clinical trials. ${ }^{12-17}$ Topical ganciclovir has been found to be safe and effective in the treatment of herpetic epithelial keratitis. A randomized prospective clinical trial was carried out in Africa and Europe. Sixty-seven patients were included in the first clinical trial in Africa, and 37 patients were included in the second clinical trial in Europe. ${ }^{12}$ The results of these two clinical trials showed no statistically significant difference between ganciclovir $0.15 \%$ gel and acyclovir $3 \%$ ophthalmic ointment in patients with herpetic epithelial keratitis. Healing was achieved within one week in $85 \%$ of patients treated with ganciclovir $0.15 \%$ gel compared with $32 \%$ of patients treated with acyclovir ointment. On the other hand, $83 \%$ of patients achieved healing within one week following ganciclovir $0.15 \%$ gel compared with $72 \%$ in patients treated with acyclovir ointment. There were fewer complaints (stinging, burning sensation, blurring of vision) in the ganciclovir group than in the acyclovir group. Patients treated with ophthalmic acyclovir ointment complained of irritation and blurring of vision. The findings of these two clinical trials support the fact that ganciclovir gel is safe and effective in the treatment of HSV epithelial keratitis, and that in this regard it is superior to acyclovir. ${ }^{12}$

Hoh et $\mathrm{al}^{18}$ studied the effects of topical ganciclovir gel and acyclovir ointment in the treatment of HSV dendritic keratitis in a multicenter, prospective, randomized clinical trial. Their findings demonstrated that topical ganciclovir ophthalmic gel was as safe and effective as acyclovir in the treatment of herpetic epithelial keratitis. There was no significant difference in adverse effects between the two treatment modalities.

Following topical application, ganciclovir has been shown to penetrate the corneal stroma and to reach the aqueous humor in therapeutic levels. ${ }^{18}$ It has been shown to be as effective as acyclovir in the treatment of herpetic keratitis in humans. ${ }^{10,18}$ The effects of topical ganciclovir $0.15 \%$ gel were studied in 16 consecutive patients with corneal grafts and previous recurrences of herpetic keratitis. ${ }^{19}$ Topical ganciclovir was found to be safe and effective in this open, non-randomized study. Furthermore, long-term topical ganciclovir afforded protection against recurrence of the disease in patients who had undergone penetrating keratoplasty. ${ }^{19}$

Patients undergoing corneal transplantation for herpetic corneal disease may be treated with topical prophylactic 
antiviral agents. Such patients have latent HSV in the trigeminal ganglion and may develop recurrence of the disease in the graft, with subsequent corneal graft failure. ${ }^{15,16,20,21}$ These patients may be given either acyclovir 3\% ophthalmic ointment or ganciclovir $0.15 \%$ gel four times daily in conjunction with topical prednisolone $1 \%$ eye drops. Ganciclovir $0.15 \%$ gel may help in the prevention of recurrences of herpetic keratitis in patients undergoing corneal transplantation. Epithelial disease improvement and healing of the ulcer can occur within one week of treatment with topical ganciclovir. ${ }^{10,18,19}$ Ganciclovir given in a gel form is easy to apply and does not cause blurring of vision, unlike acyclovir petrolatum-based ophthalmic ointment (Table 2). The drug is well tolerated and has no toxic effects on the ocular surface. Corneal tissue absorption of ganciclovir can reach therapeutic levels and be found in the aqueous humor following topical application. ${ }^{9,12}$ Figures 3 and 4 show herpetic epithelial keratitis in two patients before and one week after treatment with ganciclovir $0.15 \%$ ophthalmic gel.

Ganciclovir is an important inducer of cell death. The efficacy of ganciclovir and acyclovir in inducing apoptosis in the HSV thymidine kinase system was compared by Shaw et al. ${ }^{13}$ Treatment with ganciclovir induced rapid apoptosis of HSV-infected cells. In contrast with up to seven days' exposure, acyclovir did not induce apoptosis. Ganciclovir given topically did not cause systemic or hematologic adverse effects.

Long-term prophylaxis with ganciclovir in patients who had recurrent herpetic keratitis has not been studied. Future prospective, long-term clinical trials are needed to assess the safety and efficacy of topical ganciclovir prophylactic strategies in the prevention of recurrent herpetic corneal disease. ${ }^{19}$ Corneal transplantation for corneal scarring induced by HSV can restore vision and induce rapid recovery. Recurrence of herpetic keratitis in the transplanted graft, however, may

Table 2 Topical ganciclovir in herpetic keratitis

Good tolerance: no blurred vision, no stinging or burning sensation

Prolonged corneal contact time

Tonicity similar to tears

$\mathrm{pH}$ adjusted to a physiologic level

Sterilizable (autoclavable)

Long and stable shelf life

Potent broad spectrum antiviral agent

Selective effects on virus infected cells

Lubricant effects for corneal anesthesia

Penetration into the aqueous humor following topical application

As effective as acyclovir but at $20 \times$ less concentration

No systemic adverse effects lead to corneal graft rejection and decreased vision due to recurrence of HSV in the transplanted cornea. ${ }^{14-21}$ Therefore, it is recommended that long-term prophylaxis with topical ganciclovir gel or acyclovir ointment might help to prevent recurrence of the disease and subsequent graft rejection. It is generally agreed that corneal graft rejection and failure following corneal transplantation in patients with herpetic keratitis remains high and that the survival rate of the regraft is around $50 \%{ }^{21}$ The use of topical antiviral prophylaxis may help in the prevention of herpetic keratitis. ${ }^{15-17,19-23}$ Long-term application of ganciclovir ophthalmic gel is an effective prophylaxis against herpetic keratitis in this group of patients and appears to be a good therapeutic strategy. ${ }^{21,24-26}$ Recurrence of herpetic keratitis in the corneal graft following penetrating keratoplasty poses a therapeutic dilemma. Herpetic keratitis may lead to corneal graft rejection which requires increased use of topical corticosteroids which may aggravate the herpetic infection.

Low-dose oral acyclovir has been shown to be effective in preventing recurrent genital herpes and appears to be a safe long-term prophylactic measure. ${ }^{27-29}$ Recurrences of genital herpes are prevented by long-term, low-dose oral acyclovir. This therapeutic strategy may also be applied to recurrences of ocular herpes. Prophylaxis in patients who have undergone penetrating keratoplasty for herpetic disease may prevent corneal graft failure. Oral acyclovir does not appear to be cost-effective for the prevention of HSV eye disease. ${ }^{22}$ Topical use of ganciclovir or acyclovir ointment as prophylaxis in patients with corneal grafts may spare the use of systemic oral antiviral agents such as acyclovir. ${ }^{21}$ The good levels of ganciclovir in aqueous humor following topical application make this drug a potential therapeutic modality for the treatment and prophylaxis of herpetic keratouveitis. However, the long-term safety and efficacy of ganciclovir in the treatment of stromal keratitis and herpetic keratouveitis remain to be determined. Prospective clinical trials to assess the safety and efficacy of long-term topical ganciclovir ophthalmic gel in the prophylaxis of herpetic keratitis are needed.

Chee and Jap ${ }^{30}$ studied the effects of ganciclovir in patients with cytomegalovirus (CMV) uveitis. The CMV uveitis was diagnosed by the clinical findings and by aqueous polymerase chain reaction. The recurrence rate of anterior uveitis was significantly lower in patients who received topical ganciclovir gel than patients who were treated with systemic ganciclovir. Systemic and intravitreal ganciclovir and ganciclovir implant had good response rates but also had high recurrence rates. Ganciclovir $0.15 \%$ gel had moderate 

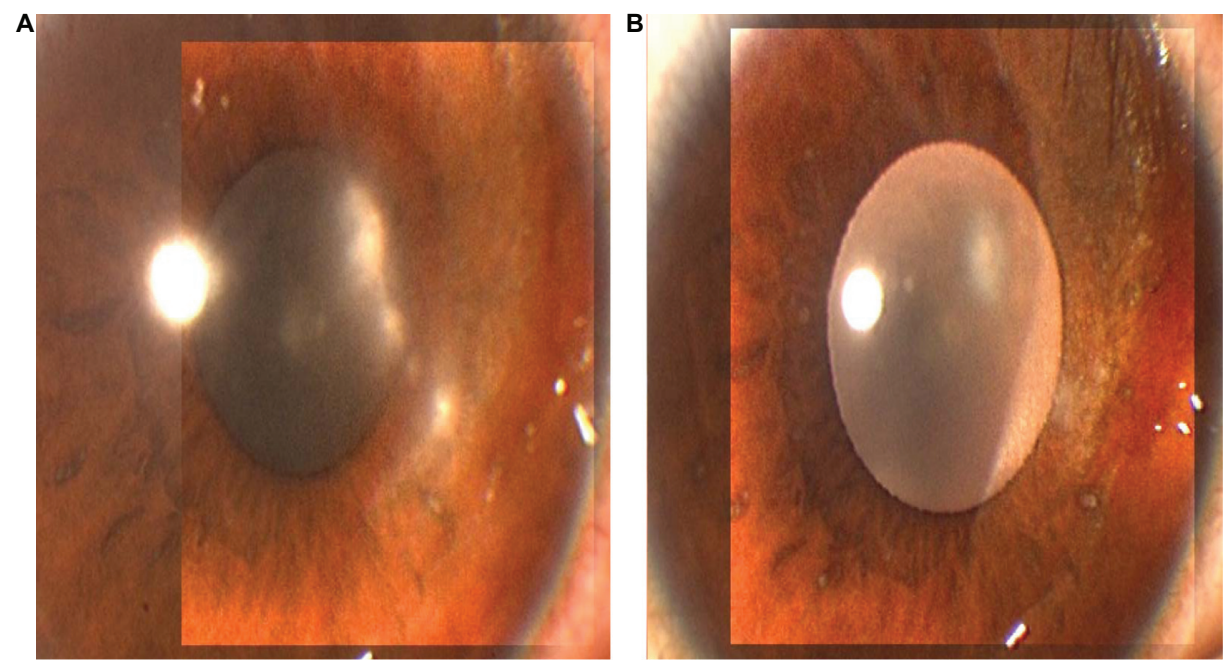

Figure 3 A) Shows the right cornea of a 12 -year-old boy with herpetic epithelial keratitis before treatment, and B) five days after treatment with topical ganciclovir $0.15 \%$ ophthalmic gel.

response rates, but its recurrence rates were also lower than those of the other treatment modalities. Topical ganciclovir $0.15 \%$ gel, therefore, may be considered for the treatment of CMV anterior uveitis.

Recent studies have focused on the ocular bioavailability of ganciclovir. In a study by Majumdar et al, ${ }^{31}$ a series of dipeptide monoester ganciclovir (GCV) prodrugs were evaluated with the goal of improving the ocular bioavailability of ganciclovir topical ophthalmic solution. Val-Val-GCV, Tyr-Val-GCV, and Gly-Val-GCV were more stable in aqueous solution than Val-GCV. All three prodrugs had much higher aqueous solubility than the current drug. Transcorneal permeability of Val-GCV and Val-Val-GCV was seven-fold to eight-fold greater than that of GCV in the presence of a proton gradient and was significantly decreased in the presence of Gly-Pro. Val-Val-GCV 1\% provided significantly
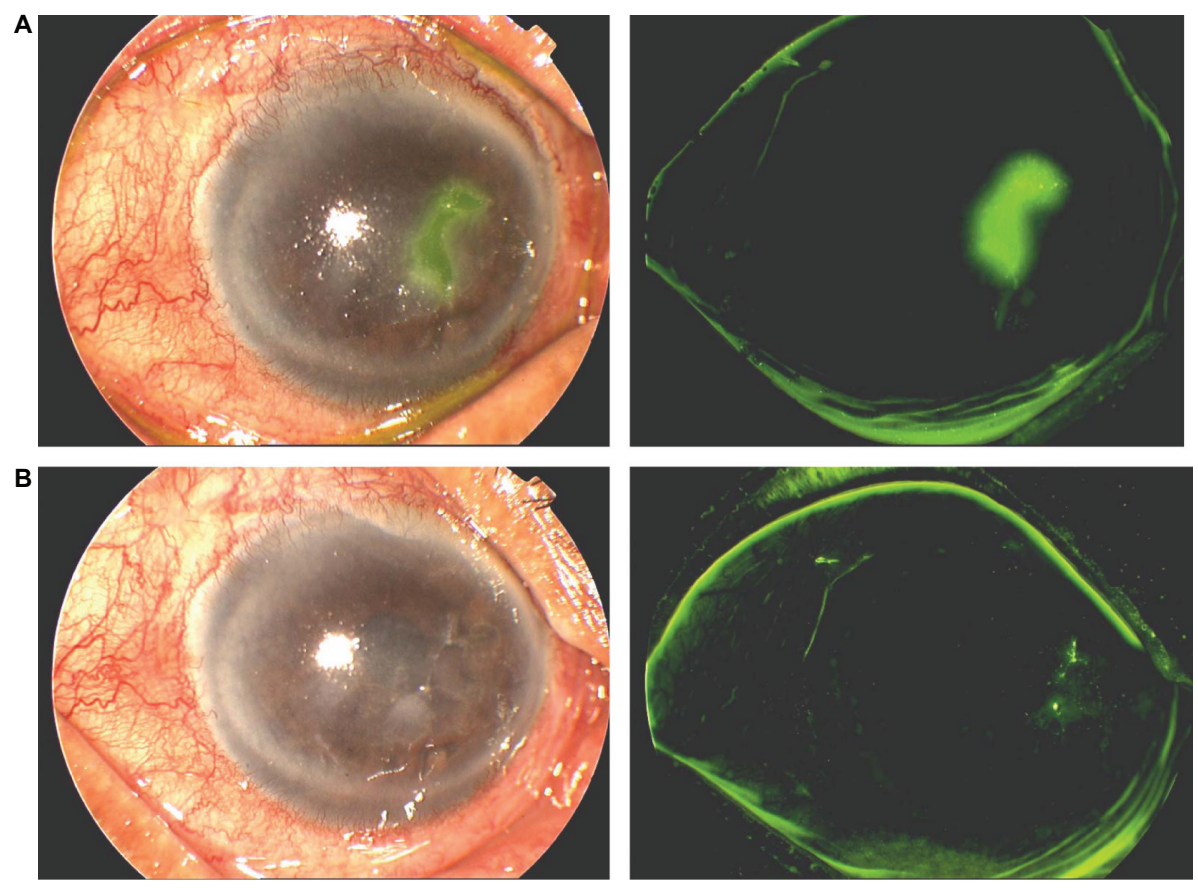

Figure 4 A) An 80-year-old male with geographic epithelial keratitis stained with fluorescein, and B) one week after treatment with ganciclovir $0.15 \%$ ophthalmic gel showing healing and re-epithelialization of the cornea. 
better therapeutic activity than trifluorothymidine against HSV-1 epithelial keratitis and was equivalent in its therapeutic activity against stromal keratitis in the rabbit eye model of herpetic keratitis. Val-Val-GCV demonstrated excellent corneal permeability and chemical stability, high aqueous solubility, and substantial in vivo antiviral activity against HSV-1.

Topical ganciclovir $0.15 \%$ gel has been shown to be as effective as acyclovir in the treatment of active herpetic epithelial keratitis. ${ }^{32,33}$ Prospective, randomized clinical trials are needed to assess the long-term safety and efficacy of topical ganciclovir in the prevention of recurrence of herpetic keratitis. Recurrent herpetic keratitis may lead to structural damage of the cornea and loss of vision. Herpes keratitis in a graft leads to rejection and graft failure. Prophylactic use of topical ganciclovir may prove to be safe in the prevention of recurrence of the disease. The intraocular penetration of topical ganciclovir in the aqueous humor may prove to be effective in the treatment of HSV keratouveitis, HSV, and CMV uveitis.

Prophylaxis may require a lower dose frequency, such as once or twice daily. The efficacy of such a therapeutic approach remains to be determined. In patients who had undergone penetrating keratoplasty for herpetic corneal scars, topical daily application of ganciclovir $0.15 \%$ gel may prove to be an effective prophylactic measure for the prevention of recurrences in the corneal graft. The dose and frequency remain to be determined. Most recurrences of HSV keratitis occur in the first year after the graft. Our patients with penetrating keratoplasty following herpetic keratitis are treated with ganciclovir $0.15 \%$ gel four times daily in the first week followed by two times daily for a period of one year. Future clinical trials are needed to assess the safety and efficacy of topical ganciclovir in the treatment and prevention of herpetic keratouveitis, and herpetic and CMV anterior uveitis.

\section{Disclosure}

The authors report no conflict of interest in this work.

\section{References}

1. Wilhelmus KR. The treatment of herpes simplex virus epithelial keratitis. Trans Am Ophthalmol Soc. 2000;98:505-532.

2. Hyndiuk R, Glasser D. Herpes simplex keratitis. In: Tabbara KF, Hyndiuk R, editors. Infections of the Eye. 2nd ed. Boston, MA: Little Brown \& Co; 1996.

3. O’Brien W. Antiviral agents. In: Tabbara K, Hyndiuk R, editors. Infections of the Eye. 2nd ed. Boston, MA: Little Brown \& Co; 1996.
4. Elftman MD, Hunzeker JT, Mellinger JC, Bonneau RH, Norbury CC, Truckenmiller ME. Stress-induced glucocorticoids at the earliest stage of herpes simplex virus-1 infection suppress subsequent antiviral immunity, implicating impaired dendritic cell function. J Immunol. 2010;184(4):1867-1875.

5. El Hayderi L, Raty L, Failla V, Caucanas M, Paurobally D, Nikkels AF. Severe herpes simplex virus type- 1 infections after dental procedures. Med Oral Patol Oral Cir Bucal. Jun 1, 2010. [Epub ahead of print].

6. Davies ME, Bondi JV, Grabowski L, Schofield TL, Field AK. 2'-nor2'deoxyguanosine is an effective therapeutic agent for treatment of experimental herpes keratitis. Antiviral Res. 1987;7(2):119-125.

7. Gordon YJ, Capone A, Sheppard J, Gordon A, Romanowski E, Araullo-Cruz T. 2'-nor-cGMP, a new cyclic derivative of 2'NDG, inhibits HSV-1 replication in vitro and the mouse keratitis model. Curr Eye Res. 1987;6(1):247-253.

8. Shiota H, Naito T, Mimura Y. Anti-herpes simplex virus (HSV) effect of 9-(1,3-dihydroxy-2-propoxymethyl) guanine (DHPG) in rabbit cornea. Curr Eye Res. 1987;6(1):241-245.

9. Castela N, Vermerie N, Chast F, et al. Ganciclovir ophthalmic gel in herpes simplex virus rabbit keratitis: Intraocular penetration and efficacy. J Ocul Pharmacol. 1994;10(2):439-451.

10. Colin J, Hoh HB, Easty DL, et al. Ganciclovir ophthalmic gel (Virgan $0.15 \%$ ) in the treatment of herpes simplex keratitis. Cornea. 1997;16(4):393-399.

11. Wilhelmus KR. Therapeutic interventions for herpes simplex virus epithelial keratitis. Cochrane Database Syst Rev. 2007;(1): CD002898.

12. Pouliquen P, Elena PP, Malecaze F, et al. Assessment of the safety and local pharamacokinetics of a $0.15 \%$ gel of ganciclovir $\left(\operatorname{Virgan}^{\circledR}\right)$ in healthy volunteers. Invest Ophthalmol Vis Sci. 1996;37 Suppl 3: S313.

13. Shaw MM, Gürr WK, Watts PA, Littler E, Field HJ. Ganciclovir and penciclovir, but not acyclovir, induce apoptosis in herpes simplex virus thymidine kinase-transformed baby hamster kidney cells. Antivir Chem Chemother. 2001;12(3):175-186.

14. Epstein RJ, Seedor JA, Dreizen NG. Penetrating keratoplasty for herpes simplex keratitis and keratoconus: Allograft rejection and survival. Ophthalmology. 1987;94(8):935-944.

15. Ficker LA, Kirkness CM, Rice NS, Steele AD. The changing management and improved prognosis for corneal grafting in herpes simplex keratitis. Ophthalmology. 1989;96(11):1587-1596.

16. Sonkin PL, Baratz KH, Frothingham R, Cobo LM. Acyclovir-resistant herpes simplex virus keratouveitis after penetrating keratoplasty. Ophthalmology. 1992;99(12):1805-1808.

17. Rapuano CJ, Cohen EJ, Brady SE, Arentsen JJ, Laibson PR. Indications for and outcomes of repeat penetrating keratoplasty. Am J Ophthalmol. 1990;109(6):689-695.

18. Hoh HB, Hurley C, Claoue C, et al. Randomized trial of ganciclovir and acyclovir in the ttreatment of herpes simplex dendritic keratitis: A multicentre study. Br J Ophthalmol. 1996;80(2):140-143.

19. Tabbara KF. Treatment of herpetic keratitis. Ophthalmology. 2005; 112(9): 1640

20. De Kesel RJ, Koppen C, Ieven M, Zeyen T. Primary graft failure caused by herpes simplex virus type 1. Cornea. 2001;20(2):187-190.

21. Tabbara KF. Pharmacologic strategies in the prevention and treatment of corneal transplant rejection. Int Ophthalmol. 2008;28(3): $223-232$.

22. Lairson DR, Begley CE, Reynolds TF, Wilhelmus KR. Prevention of herpes simplex virus eye disease: A cost effectiveness analysis. Arch Ophthalmol. 2003;121(1):108-112.

23. Akova YA, Onat M, Dauman S. Efficacy of low dose and long term oral acyclovir therapy after penetrating keratoplasty for herpes simplex keratitis. Ocul Immunol Inflamm. 1999;7(1):51-60.

24. Barron BA, Gee L, Hauck WW, et al. Herpetic Eye Disease Study. A controlled trial of oral acyclovir for herpes simplex stromal keratitis. Ophthalmology. 1994;101(12):1871-1882. 
25. Herpetic Eye Disease Study Group. Acyclovir for the prevention of recurrent herpes simplex virus eye disease. $N$ Engl J Med. 1998;339(5):300-306.

26. Herpetic Eye Disease Study Group. Oral acyclovir for herpes simplex virus eye disease: Effect on prevention of epithelial keratitis and stromal keratitis. Arch Ophthalmol. 2000;118(8):1030-1036.

27. Goldberg LH, Kaufman RH, Kurtz TO. Continuous five-year treatment of patients with frequently recurring genital herpes simplex virus infection with acyclovir. J Med Virol. 1993;Suppl 1: $45-50$.

28. Mertz GJ, Eron L, Kaufman R, et al. Prolonged continuous versus intermittent oral acyclovir treatment in normal adults with frequently recurring genital herpes simplex virus infection. Am J Med. 1988;85(2A):14-19.
29. Fife KH, Crumpacker CS, Mertz GJ, Hill EL, Boone GS. Recurrence and resistance patterns of herpes simplex virus following cessation of more than or equals to 6 years of chronic suppression with acyclovir. Acyclovir Study Group. J Infect Dis. 1994;169(6):1338-1341.

30. Chee SP, Jap A. Cytomegalovirus anterior uveitis: outcome of treatment. Br J Ophthalmol. 2010 Jun 24. [Epub ahead of print].

31. Majumdar S, Nashed YE, Patel K, et al. Dipeptide monoester ganciclovir prodrugs for treating HSV-1-induced corneal epithelial and stromal keratitis: In vitro and in vivo evaluations. $J$ Ocul Pharmacol Ther. 2005;21(6):463-474.

32. Colin J. Ganciclovir ophthalmic gel, $0.15 \%$ : A valuable tool for treating ocular herpes. Clin Ophthalmol. 2007;1(4):441-453.

33. Labetoulle M. The latest in herpes simplex keratitis therapy. $J$ Fr Ophtalmol. 2004;27(5):547-557. [in French].
Clinical Ophthalmology

\section{Publish your work in this journal}

Clinical Ophthalmology is an international, peer-reviewed journal covering all subspecialties within ophthalmology. Key topics include: Optometry; Visual science; Pharmacology and drug therapy in eye diseases; Basic Sciences; Primary and Secondary eye care; Patient Safety and Quality of Care Improvements. This journal is indexed on

Submit your manuscript here: http://www.dovepress.com/clinical-ophthalmology-journal

\section{Dovepress}

PubMed Central and CAS, and is the official journal of The Society of Clinical Ophthalmology (SCO). The manuscript management system is completely online and includes a very quick and fair peer-review system, which is all easy to use. Visit http://www.dovepress.com/ testimonials.php to read real quotes from published authors. 